\title{
Formal Microeconomic Foundations and the First Welfare Theorem
}

\author{
Cezary Kaliszyk \\ University of Innsbruck \\ Department of Computer Science \\ Innsbruck, Austria \\ cezary.kaliszyk@uibk.ac.at
}

\author{
Julian Parsert \\ University of Innsbruck \\ Department of Computer Science \\ Innsbruck, Austria \\ julian.parsert@uibk.ac.at
}

\begin{abstract}
Economic activity has always been a fundamental part of society. With recent social and political changes economics has gained even more influence on our lives. In this paper we formalize two economic models in Isabelle/HOL: the pure exchange economy, where the only economic actors are consumers, as well as a version of the Arrow-Debreu Model, a private ownership economy, which includes production facilities. Interestingly, the definitions of various components of the economic models differ in the economic literature. We therefore show the equivalences and implications between various presentations, which allows us to create an extensible foundation for formalizing microeconomics and game theory compatible with multiple economic theories. We prove the First Theorem of Welfare Economics in both economic models. The theorem is the mathematical formulation of Adam Smith's famous invisible hand and states that a group of self-interested and rational actors will eventually achieve an efficient allocation of goods. The formal proofs allow us to find more precise assumptions than those found in the economic literature.
\end{abstract}

\section{CCS Concepts - Theory of computation $\rightarrow$ Logic and verification;}

Keywords Isabelle, First Welfare Theorem, Microeconomics, Game Theory

\section{ACM Reference Format:}

Cezary Kaliszyk and Julian Parsert. 2018. Formal Microeconomic Foundations and the First Welfare Theorem. In Proceedings of 7th ACM SIGPLAN International Conference on Certified Programs and Proofs (CPP'18). ACM, New York, NY, USA, 11 pages. https://doi. org $/ 10.1145 / 3167100$

\footnotetext{
Permission to make digital or hard copies of part or all of this work for personal or classroom use is granted without fee provided that copies are not made or distributed for profit or commercial advantage and that copies bear this notice and the full citation on the first page. Copyrights for thirdparty components of this work must be honored. For all other uses, contact the owner/author(s).

CPP'18, January 8-9, 2018, Los Angeles, CA, USA

(c) 2018 Copyright held by the owner/author(s).

ACM ISBN 978-1-4503-5586-5/18/01.

https://doi.org/10.1145/3167100
}

\section{Introduction}

Attempts at describing economics and human behavior have been made throughout history by people ranging from political science to philosophy. The most famous representative thereof is Smith who, in the 18th century, described several findings such as the invisible hand, in his book "An Inquiry Into the Nature and Causes of the Wealth of Nations" [26]. At the turn of the last century economics became more and more formal. Leading to the foundations of the mathematical field of Game Theory and related fields by von Neumann and Morgenstern [32]. This lead to famous mathematical results such as Arrows Impossibility theorem or the Vickrey-ClarkeGroves (VCG) auction mechanism [22]. Lately Algorithmic Game Theory and Mechanism Design have also gained interest in the field of Computer Science [3].

Economic models and mathematical analysis and reasoning have become standard practice for policy decisions. These methods are also employed by market authorities, as well as financial institutions. Even corporations such as Google or Yahoo utilize game theoretic results by using auction mechanisms to sell advertising space on search results. Interestingly, Google thought that they were using a generalized version of the VCG auction to sell advertising. However, it later was shown that their mechanism was flawed and thus could not exhibit desired properties [8]. Since the 2007/2008 financial crisis multiple critiques of financial models and their applicability have emerged [17, 29]. With the growing power of interactive theorem proving systems [13], we believe they can be used to more formally and clearly specify economic theory. This allows us to better understand the assumptions necessary and therefore the limitations of the theory.

\subsection{Contributions}

We formalized the basics of microeconomics and game theory, specified two economic models, and formalized certain results in these models. We also showed the definitions of various microeconomic concepts originating from multiple sources across the literature equivalent. In particular:

We formalized the preference relations and properties thereof. We defined properties such as local non-satiation, convexity and monotonicity. To remove ambiguities we proved that the different definitions of local non-satiation found in 
the books $[11,18,20]$ are equivalent. We also considered convexity and proved alternative definitions found in $[1,11]$ equivalent. We defined ordinal utility functions and showed their existence under precise assumption.

Using these definitions we formalized two market models: a version of the Arrow-Debreu Model and the pure exchange economy model. Finally, we prove that Walras' law, as well as the First Welfare Theorem hold in both models. Furthermore, we were able to reduce the set of assumptions for each theorem refining some of the results from the economics literature.

\subsection{Related Work}

There have been multiple attempts at formalizing economical concepts. The ForMaRE project [19] intended to apply formal mathematical reasoning to economic results. One way of doing so, was the development of the "Auction Theory Toolbox" [16]. Furthermore, some theories in stochastic finance have been formalized by Jäger [14]. More recently Echenim and Peltier [7] formalized an option pricing model due to [5].

Interestingly, there have been very few formalizations of results from Game Theory. One of the few examples is Le Roux's [25] formalization of Nash Equilibria in Coq. In Social Choice Theory, another mathematical area with applications to economics, Wiedijk [34] formalized Arrow's Impossibility Theorem using Mizar. The same theorem was also formalized by Nipkow in Isabelle [21]. Furthermore, Gammie [9] formalized May's Theorem and Sen's Theorem as well as other Social Choice Theory results. Finally, Eberl [6] formalized multiple basics of Randomised Social Choice Theory.

To the best of our knowledge there have been no attempts to formalize microeconomics. Although, it is closely related to game theory and social choice theory, we found that the aforementioned formalizations had only little in common with the part of microeconomics that we intended to formalize. This includes various definitions and lemmas leading to the First Welfare Theorem.

\subsection{Content}

In Section 2 we will will give a brief overview of Isabelle and introduce notation and syntax that we use. In Section 3 we introduce microeconomic notions and terminology that will be used later, as well as examples and a graphical interpretation of the topic. In Section 4 we introduce precise definitions as used in the Isabelle/HOL formalization. In addition to preferences, utility functions, we also define the considered models. Then, in Section 6, we derive the lemmas needed to prove the First Welfare Theorem, show an outline of the proof of the main theorem, and discuss differences across the considered microeconomic models.

\section{Isabelle/HOL and Notations}

We give a brief introduction to Isabelle/HOL, which is the tool we used for the formalization, focusing on locales as we use these extensively. In addition we introduce the notations used in the formalization.

Isabelle/HOL [33] is an Interactive Theorem Prover based on higher-order logic. Hence, terms are typed, and types will be denoted by Greek letters $\beta, \gamma, \ldots$ In addition Isabelle/HOL provides a type class mechanism [12] similar to that of Haskell. Type classes allow constraining types to those that fulfill certain properties. For example: The term $t$ of type $\gamma::$ ordered_euclidean_space is polymorphic, however the type class implies that $t$ adheres to the euclidean axioms and has some notion of order. In this specific case this order is the componentwise order. We will use Isabelle/HOL standard library type constructors including real for real numbers, $\gamma \Rightarrow \beta$ for functions, $\gamma$ set for sets, and $\gamma \times \beta$ for products. In addition we let $\gamma$ relation denote the type $(\gamma \times \gamma)$ set that is, the type of sets of pairs.

Further, we will use the symbol $\geq$ to denote a preference relation. In addition we will use $>$ and $\approx$ to indicate strict and indifferent preferences respectively. In the formalization and in Isabelle code the more precise notation $x \geq[R] y$ is used, indicating the pair $(x, y)$ is an element of $R$. Furthermore, the implicit argument $i$ to symbols such as $\geq_{i}, U_{i}$ are used in environments, where a preference $\geq$ etc. can be assigned to more than one entity. Because of the limitations of Isabelle's subscript arguments we use the [_] notation instead. Hence, $\operatorname{Pr}[i], U[i], \mathcal{E}[i]$ etc. denote the application of the function $\operatorname{Pr}, U, \mathcal{E}$ to the argument $i$. Similarly, $\Theta[i, j]$ is the (curried) application of the function $\Theta$ to $i$ and $j$. For instance the term $x \geq[\operatorname{Pr}[i]] y$ indicates that the pair $(x, y)$ is in the relation $\operatorname{Pr}[i]$, which is the relation obtained after applying $i$ to $\operatorname{Pr}$.

The inner product of two elements $x$ and $y$ which adhere to the type class real_inner, is denoted by $\bullet$. The function arg-max-set applied to the function $f$ and the set $S$, defines the set of all largest elements in the image of $S$ under $f$, with respect to a fixed order.

\subsection{Locales}

Locales [2] are one of the Isabelle mechanisms providing a module system. Their behavior corresponds to that of a context in which certain terms and assumptions are fixed. In particular, a theorem $T$ proven within a locale context $C$ can be seen as a derivation of $T$ given the premises and quantified terms specified in $C$. In addition, locales also define a predicate of the same name that combines the assumptions. This means that one can show that one locale (i.e., definition) implies an other locale (i.e., definition). This can be done using sublocale $A B$, which requires a proof of $A \rightarrow B$. With this, all theorems proven in the context of locale $B$ become also available in the locale $A$ without additional assumptions. 


\section{Market and Economy}

In this section we will give a brief overview of Microeconomics. Furthermore, we will introduce some terms that we will use later on and give some intuition as to where in the vast field of economics we are located.

When talking about an economy, we mostly refer to the economy of a specific country or area. Generally speaking, an economy is a set or collection of markets, which will be explained subsequently. We can differentiate between different economic units according to their functions. The units we are interested in fall into two groups, buyers and sellers. The former includes consumers, who purchase goods and services, the latter includes producers, which sell produced goods and services. It should be apparent, that even though we make this distinction, economic actors (i.e., units) can fall into both categories. For instance, consumers usually buy goods, as well as offer labor, on the other hand, firms require resources to produce goods which later can be sold. Both are examples where the same entity acts as a supplying (i.e., producer) and as a demanding (i.e., consumer) agent. However, we can think of agents as being buyers or consumers when they are buying something and sellers or producers when they are selling something. With that in mind, we can define a market as being a set of buyers and sellers trading goods and services with prices that are determined through their interaction [24].

We will consider a special case of a market economy, more specifically a model with perfect competition. A market has perfect competition, if each agent takes prices as given. More precisely, prices are independent of each agent's action. This rules out the existence of monopolies, oligopolies, etc. Competitive markets can be observed in branches, where each producer and consumer is only responsible for a small fraction of the total amount of production and consump$\operatorname{tion}^{1}$ [31].

Whether it is voting on a candidate or playing a game of chess, a player or actor is in a situation, where a choice needs to be made. Be it, which move to make next or whom to vote for on a ballot. These problems have the same underlying structure. A subject is faced with a choice and each choice will have an outcome. In addition, each player is able to rank these outcomes. This introduces three features that a decision problem for a single actor requires: Actions, Outcomes, Preferences [30]. Our focus will be the behavior of economic actors (i.e., units) and the result of their actions in an economy. These actions include trades (exchanges), choices etc., whereas properties can include efficiency of allocations or equilibrium states.

\footnotetext{
${ }^{1}$ The standard example for a competitive market in reality is the market for wheat. Here, each farmer is only responsible for a small fraction of wheat produced, and each consumer is only responsible for a small fraction of wheat bought.
}

To sum it all up, we consider the game of a market. This market has multiple players which can fall into two categories, consumers and producers. The actions a player can perform are trades. Each actor can trade goods and services for other goods and services for a certain price. The outcomes are simply the assets owned after the trade. A set of assets is referred to as consumption set. These consumption sets can be ranked with respect to each other according to an agents personal taste. We call these relations preference relations. Each economic agent has a separate preference relation. Thus two agents might reach a preferable outcome by trading with each other. We will introduce definitions and apply formal reasoning on these concepts in subsequent sections.

\section{Formal Microeconomic Preliminaries}

In the previous section we introduced some microeconomic and game theoretic concepts in an informal manner. In this section we present their precise formal definitions and some of their most basic properties as formalized in Isabelle/HOL.

\subsection{Preference and Utility}

First, we introduce preference relations. Generally, these define a binary relation between pairs of outcomes (in our case, consumption bundles). This relation is supposed to represent an agent's personal taste and enables us to compare two bundles with each other. In mathematical terms a rational preference relation corresponds to a total preorder. Hence for any two bundles $a$ and $b$ one and only one of $a>b$ (i.e., $a$ is strictly preferred to $b$ ), $b>a$ or $a \approx b$ (i.e., the agent is indifferent to $a$ and $b$ ) must hold. We formalize preference relations as transitive and reflexive binary relations following considered literature. We followed set-theoretic methods for defining binary relations, based on the experiences by Caminati et al. in the ForMaRE project [4], which in case of our formalizations allows staying closer to the literature. The definition of rational preference relation on a set carrier is presented in Fig. 1. The relation is simply a set of pairs that constitutes a total preorder on the carrier. In addition, we assume, that no element in the relation is outside of the carrier set. Since we intend to stay as general as possible to enable later use of this formalization, we do not restrict the underlying type $\delta$.

However, in practice preference relations are rarely used. This is due to the fact that mathematical techniques (e.g., optimization, representation, ...) are easier on a numerical representation of preference relations [15]. Hence, in practice the use of utility functions is more common.

Definition 1 (Utility Function). A utility function $u: X \mapsto$ $\mathbb{R}$ where $X$ is the commodity space, is said to represent the preference relation $\geq$ if

$$
\forall x y \cdot x \geq y \Longleftrightarrow u(x) \geq u(y) .
$$




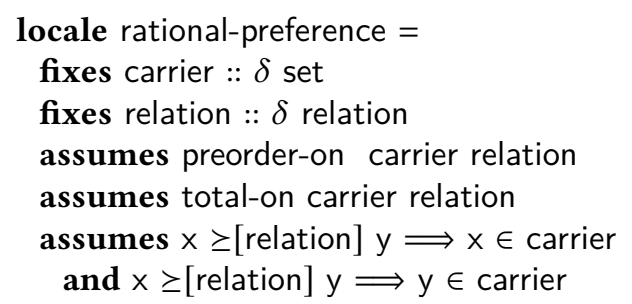

Figure 1. We use predefined predicates preorder-on and totalon to achieve our goal. The third assumption constitutes, that no element in the relation is outside of the carrier set.

This definition translates nicely to a formal counterpart as shown in Figure 2. For both preferences and utility functions

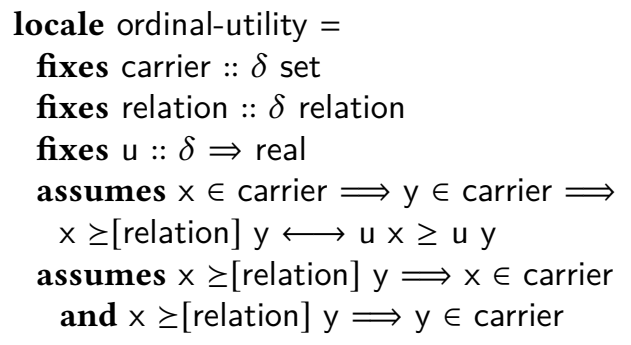

Figure 2. The first assumption creates the relation between a preference relation (relation) and a utility function $(u)$ as described in Definition 1.

we also need definitions and lemmas with restrictions on the type $\delta$. Using type classes (cf. Section 2) we introduce the sublocale eucl-ordinal-utility which requires the type $\delta$ to adhere to the axioms of euclidean space. However, even in the unrestricted general case (Figure 2), one can notice how the assumptions necessary for defining preference relations (Figure 1) are omitted. Nevertheless, we can show that the assumptions still imply that relation and carrier in the context described in Figure 2 still constitute a total preorder on the carrier set. This allows us to show that all lemmas that hold in the context of rational preferences must also hold with utility functions:

\section{sublocale ordinal-utility $\subseteq$ rational-preference carrier relation}

Furthermore, utility functions do not contain more information than preference relations. They are merely a different representation of the same information. These so called ordinal utility functions only convey a meaning when comparing values to other values [18]. That is, if $u(b):=10$ and $u(c):=30$ the only information that can be inferred is that $c$ is in fact strictly preferred to $b$, while the values $(10,30, \ldots)$ do not represent intensity or strength of the preference [15]. In fact, as Lemma 1 shows, utility functions are only unique up to a monotonic transformation.
Lemma 1 (ordinality-of-utility-function).

fixes $f::$ real $\Rightarrow$ real

assumes monotone $(o p>)(o p>) f$

shows $(f \circ u) x>(f \circ u) y \longleftrightarrow u x>u y$

\subsection{Further Definitions}

We now introduce further concepts in terms of preferences and utility. Interestingly, while conducting research in existing literature, we discovered that books and publications differed in the definitions, making it unclear whether the different sources are comparable. We decided to eliminate any ambiguities by considering all definitions, formalizing them, and proving them equivalent within Isabelle. This allows us to reason across multiple sources in literature, as well as to solidify the foundations of our formalization.

Concepts such as convexity or continuity also exist when considering utility functions and preferences, however we will focus on local non-satiation, as this is a crucial assumption for our final result.

Definition 2 (Local non-satiation). Preferences are said to be locally non-satiated, if for every consumption bundle there exists a strictly preferred bundle within any proximity.

Local non-satiation is a crucial assumption for the First Welfare Theorem. Given a preference relation $R$ over a set $S$, local non-satiation allows us to put maximization of $R$ (or its respective utility function $u$ ) and closed/openness of $S$ into relation:

1. If $S$ is open, then $u$ has no maximum.

2. If $S$ is closed, then the maximum of $u$ is in the bound of $S$.

The formal Definition 3 corresponds to the most common definition (see for example Mas-Colell [20]).

Definition 3 (local-nonsatiation).

local-nonsatiation $\mathrm{B} \mathrm{P}=$

$(\forall \mathrm{x} \in \mathrm{B} . \forall \mathrm{e}>0 . \exists \mathrm{y} \in \mathrm{B}$. norm $(\mathrm{y}-\mathrm{x}) \leq \mathrm{e} \wedge \mathrm{y}>[\mathrm{P}] \mathrm{x})$

Note that although the types of $B$ and $P$ are polymorphic, using the type class mechanism we can ensure that some function is defined for elements in $B$ that satisfies the properties of a norm without explicitly constructing it.

An alternative definition of local non-satiation is due to Jehle and Reny [11], which uses the notion of ball $l_{e}$. This can be shown to be equivalent using light automation. Some sources, such as [27] even omit local non-satiation entirely. Instead the notion of monotone preferences is used. These preferences imply that given an element $x$ all elements $y$ such that $y>x$ are strictly preferred to $x$. More concisely, $x>y \rightarrow x>y$. In order to formalize and use results from these theories we prove Lemma 2. Thus confirming that under the condition that the consumption set is an up-set, monotonicity implies local non-satiation. Defining monotone preferences requires an order on vectors, which we did not require for local non-satiation. Thus we use the type 
class ordered_euclidean_space which defines the componentwise order on top of the euclidean axioms. We will omit type class information in the remainder of the paper wherever it is clear from context.

Lemma 2 (unbounded-above-mono-imp-lns).

assumes $\forall a \in$ carrier. $(\forall x>a . x \in$ carrier $)$

assumes monotone-preference carrier relation

shows local-nonsatiation carrier relation

Since our goal was to stay as general as possible in all cases, we proceeded to use local non-satiation when possible. However, adding corollaries proving that these results also hold under monotonicity, is trivial using Lemma 2.

As one can imagine in our models - much like in reality agents cannot spend more than what is available to them. In particular they are bound by a budget constraint or budget set. Definition 4 describes how such a budget set is calculated.

Definition 4 (budget-constraint).

budget-constraint P S W $=\{\mathrm{x} \in \mathrm{S}$. P $\bullet \mathrm{x} \leq \mathrm{W}\}$

The set of affordable bundles (i.e., budget set) is comprised of all bundles of the consumption set $S$, where the monetary value of the bundle does not exceed the wealth $W$. We will introduce consumption sets in the subsequent section. Intuitively, values of bundles and wealth are real values which constitute a monetary value. The wealth is calculated differently in each economic model. Hence, we will therefore return to $W$ calculation in Section 5. The value of a bundle $x$ is simply the inner product of a price vector $P$ with $x$.

\section{Model Construction}

Up to this point we considered general concepts, which are also used in Game Theory or Social Choice Theory. In addition, these concepts only concern themselves with each agent individually and are independent of the market models. However, since one of our goals is to formalize the First Welfare Theorem, we need to define a market model to which the theorem applies. In particular we will consider two market economies, the pure exchange economy and a private ownership economy. The latter is comparable to the ArrowDebreu-Model [10].

First, we need to describe objects that can be owned, traded or consumed by economic units. In Sections 4 and 4.2 we referred to these as consumption bundles or even more abstractly outcomes. Indeed the term consumption bundle is already specific to our use of these concepts. Since we consider General Equilibrium Theory we consider more than just one commodity. Consumption bundles describe multisets of commodities. The most common representation (and the one we use) are vectors. Each component of a consumption vector describes the amount of that commodity. This allows us to use the previously introduced notions of inner product, norm etc. in a natural way.
Further, we introduce the notion of consumption sets. This simply describes the set of possible consumption bundles (i.e., vectors). For instance, consider a debt free economy. In this case the consumption set will be the set of all non negative vectors (vectors with non negative components). An additional example is a consumer that can only consume 50 apples, while only being able to afford 20. This consumption set would include all elements between 0 and 50 . Generally speaking most literature puts restrictions that are not always necessary on production and consumption sets. Thanks to the formalization we were able to omit all such unnecessary restrictions.

Next, we need a mapping that assigns ownership of commodities to agents. This is also known as an allocation ${ }^{2}$. The first allocation in an economy is also called initial endowment, which specifies each agent's consumption bundle before any trade takes place. When looking at allocations, one can observe their efficiency, attainability, or fairness. We are only interested in efficiency and feasibility (i.e., attainability). The latter of which will be dealt with shortly. To define efficiency, we introduce an ordering on allocations:

Definition 5 (Pareto ordering). An allocation $X$ Pareto dominates $Y$ (i.e., $X$ is higher than $Y$ in the induced Pareto ordering) if and only if $X$ does not decrease any agents utility compared to $Y$, while simultaneously increasing the utility of at least one agent.

Thus, we say an allocation $X$ is more wasteful (i.e., less efficient) than $Y$ if $Y$ Pareto dominates $X$. This translates to the formal Definition 6.

Definition 6 (pareto-dominating).

$\mathrm{X}>$ Pareto $\mathrm{Y} \longleftrightarrow$

$(\forall \mathrm{i} \in$ agents. $\mathrm{U}[\mathrm{i}](\mathrm{X} \mathrm{i}) \geq \mathrm{U}[\mathrm{i}](\mathrm{Y} \mathrm{i})) \wedge$

$(\exists \mathrm{i} \in$ agents. U[i] $(\mathrm{X} \mathrm{i})>\mathrm{U}[\mathrm{i}](\mathrm{Y} \mathrm{i}))$

This is comparable to a pointwise comparison. As stated in Definition 5, $X$ cannot be a decrease for any agent, while being an increase for at least one agent.

Given this ordering we say that an allocation is Pareto Optimal if there exists no allocation, that is both Pareto dominating and feasible. Feasibility (i.e., attainability) describes the limiting factor in our economic models. An allocation is feasible if the sum of the goods allocated (i.e., consumed) does not exceed the sum of goods available (i.e, produced). Our final result also requires the concept of the Walrasian Equilibrium (also called the Competitive Equilibrium). Its exact definition differs across economic settings, therefore we will introduce it only within their respective market models. However, we can define the more abstract notion of consumer problem. Simply put, the consumer problem describes the optimization problem a consumer faces, when being tasked with choosing a consumption bundle. Technically speaking a

${ }^{2}$ Compare an operating system assigning or allocating chunks of memory to processes. 
consumer seeks to maximize the utility function while being subject to a budget constraint. However, feasibility, budget, and equilibria are definitions dependent on the model in consideration. Therefore, we will be introducing these definitions within their respective contexts, the exchange economy, and the private ownership economy.

\subsection{Exchange Economy}

In an exchange economy the only economic actors are consumers. These consumers are merely equipped with an initial endowment. Hence, initially each agent owns a single, possibly empty, bundle, which then can be traded with other agents. These trades correspond to the actions in the consumer problem with the limiting factor being the initial endowment. Hence, the initial wealth ${ }^{3}$ of an agent cannot be surpassed. In other words, bundles that cannot be afforded by a consumer, cannot be chosen by a consumer, therefore imposing a boundary on the options a consumer has. This allows us to define the allocations to be feasible if and only the sum of goods does not exceed the sum of the goods in the initial endowment. And since we lack any kind of production capability, defining the Walrasian Equilibrium is straightforward. An allocation together with a price vector $P$, is said to form a Walrasian Equilibrium, if every agent maximizes their respective utility function with respect to their set of affordable bundles (referred to as budget set in economics literature). In the remainder of this section we will focus on the formal definitions of these concepts.

Once more we use the Isabelle module system to describe the environment of an exchange economy (Figure 3). First, we have a finite nonempty set of individuals (agents). All agents have the same consumption set. In this simple model, we define the consumption set to be the set of all elements of type $\delta$. Hence the predicate pre-arrow-debreu-cons-set in the second assumption. For readability and brevity we will abbreviate consumption-set with CS. In addition we have three functions, each mapping every agent $i$ to their respective, initial endowment $\mathcal{E}_{i}$, preference relation $P r_{i}$, and utility function $U_{i}$. It follows that, much like the initial endowment $\mathcal{E}$, an allocation is simply a function of type $\beta \Rightarrow \delta$, assigning to each individual $\beta$ in agents a bundle of type $\delta$. The function $U$ applied to an individual $i$ returns a utility function of type $\delta \Rightarrow$ real that represents the preference relation $\operatorname{Pr}[i]$. This is ensured with the third assumption. Finally, we fix a strictly positive price vector. From now on will refer to a price vector that adheres to this condition with Price while $P$ will simply denote an arbitrary price vector.

Next, we define the notion of feasibility. As previously stated, an allocation is feasible if the sum of goods allocated does not exceed the sum of goods available. Considering that

\footnotetext{
${ }^{3}$ Wealth of an agent, is the value of the owned consumption bundle, which is the sum of all goods owned multiplied with the price of each good. Further details will be discussed in Section 4 .
}

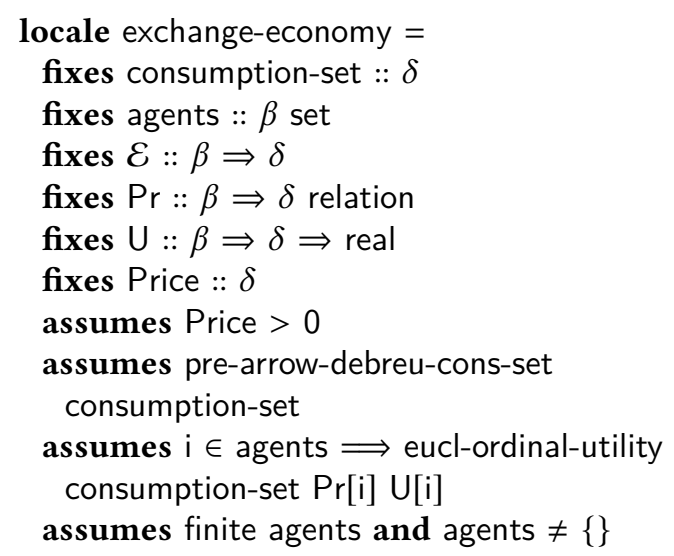

Figure 3. The module for an exchange economy with all constants and assumptions.

we are in an exchange economy with no production facilities, the goods available are exactly the goods allocated with the initial endowment. This gives us Definition 7, where given an allocation $A$ and an endowment $E$, the pointwise sum is calculated and compared.

Definition 7 (feasible-allocation).

feasible-allocation $\mathrm{A} \mathrm{E} \longleftrightarrow$

$\left(\sum \mathrm{i} \in\right.$ agents. $\left.\mathrm{A} \mathrm{i}\right) \leq\left(\sum \mathrm{i} \in\right.$ agents. $\left.\mathrm{E} \mathrm{i}\right)$

Next, we can define the Walrasian Equilibrium. An equilibrium is reached once every agent solves the consumer optimization problem. The solution to this maximization problem can be described as follows:

The bundle $x_{i}$ is a solution to the consumer problem of agent $i$ if

$$
x_{i} \in \underset{x}{\arg -\max } U_{i}(x)
$$

subject to $x \in$ budget-constraint $P E_{i}$.

A competitive equilibrium comprises a feasible allocation $\mathrm{A}$, a price $\mathrm{P}$, and an endowment $\mathrm{E}$, with the latter two required to calculate the budget set. This results in Definition 8 where $\mathrm{U}[\mathrm{i}], \mathrm{E} \mathrm{i}$, and $\mathrm{X}$ i represent $U_{i}, E_{i}$, and $x_{i}$ respectively.

Definition 8 (comp-equilib-endow).

comp-equilib-endow P A E $\longleftrightarrow$ feasible-allocation A E $\wedge$ $(\forall \mathrm{i} \in$ agents. $\mathrm{X} \mathrm{i} \in \arg$-max-set $\mathrm{U}[\mathrm{i}]$

(budget-constraint P CS (P• E i)))

Finally, we define Pareto Efficiency as a combination of the previously defined feasibility and Pareto ordering.

Definition 9 (pareto-optimal-endow).

pareto-optimal-endow X E feasible-allocation X E $\wedge$ ( $\nexists X^{\prime}$. feasible-allocation $X^{\prime} \mathrm{E} \wedge \mathrm{X}^{\prime}>$ Pareto $\mathrm{X}$ )

A feasible allocation that is not Pareto dominated by any feasible allocation is Pareto Optimal. With Definitions 7 to 9 we introduced all prerequisites necessary for the First Welfare Theorem in an exchange economy. 


\subsection{Private Ownership Economy}

As the second considered economic model we took the ArrowDebreu model [10] as a guideline. However, during the formalization process we realized that for many lemmas, including even the First Welfare Theorem itself, certain assumptions are redundant. These assumptions include restrictions such as convexity, etc. on both consumption and production sets. These restrictions are most likely due to the fact that the final goal in some sources is proving the existence of an equilibrium in this model. However, since interactive theorem proving tools allow us to dynamically included assumptions precisely when necessary we only used the least amount of restriction necessary and refer to this model as "Pre-Arrow-Debreu" model.

In contrast to the previous model, the private ownership economy has production facilities called firms. Each firm has technologies which define the relation between input and output (cf. Example 1) These firms are entirely owned by agents, who share the profits ${ }^{4}$ of the firm according to their share of the firm (cf. shareholders in the traditional sense). Therefore the wealth of each agent is comprised of the initial endowment and the sum of all profits of owned firms. Thus the budget set of a consumer is the sum of the returns of firms and the initial endowment. The consumer problem remains the same as in the exchange economy, albeit with a different budget set. In addition, we need to consider the problem a producer is facing. Similar to the consumer problem, the producer problem is also a maximization problem. In particular a firm seeks to maximize its profit over the so called production set. These production sets describe the production possibilities of companies. Example 1 gives a simple production set.

Example 1 (Production set). Imagine a firm that produces apple juice. We require inputs such as apples, sugar and water to produce the output that is apple juice. If we assume that our technology enables us to produce 2 units of juice using 1 unit of apples, 2 units of water and 1 unit of sugar then we say that the vector $(2,-1,-2,-1)$ is part of this company's production set $\operatorname{Pr}$. By adding further assumptions such as scalability up to a factor $k$ we can say that $\forall a \leq$ $k$. $(2,-1,-2,-1) a \in P$. Another common assumption, is that there are no production bundles strictly greater than 0 in the set, since that would imply the possibility of creating something out of nothing.

The combination of feasibility, the utility maximization of consumers, and the profit maximization of producers, and a certain price, defines the Walrasian Equilibrium in the private ownership economy. In other words, each economic actor is purely self interested and seeks nothing but their own optimum (i.e., maximal utility or profit).

\footnotetext{
${ }^{4}$ Since by definition the zero vector is in the production set, firms will never incur a loss.
}

Formally we once again create a context which collects all the mappings and assumptions necessary (Figure 4). Now this also includes a set of firms and a production set for each firm. Therefore, we include an additional mapping $\Theta$, which maps an individual $i$ to the share of a firm $j$. That is, if individual $i$ owns $50 \%$ of company $j$ then $\Theta[i, j]=0.5$. In addition, the third assumption guarantees that every firm is entirely owned by agents. In an exchange economy the

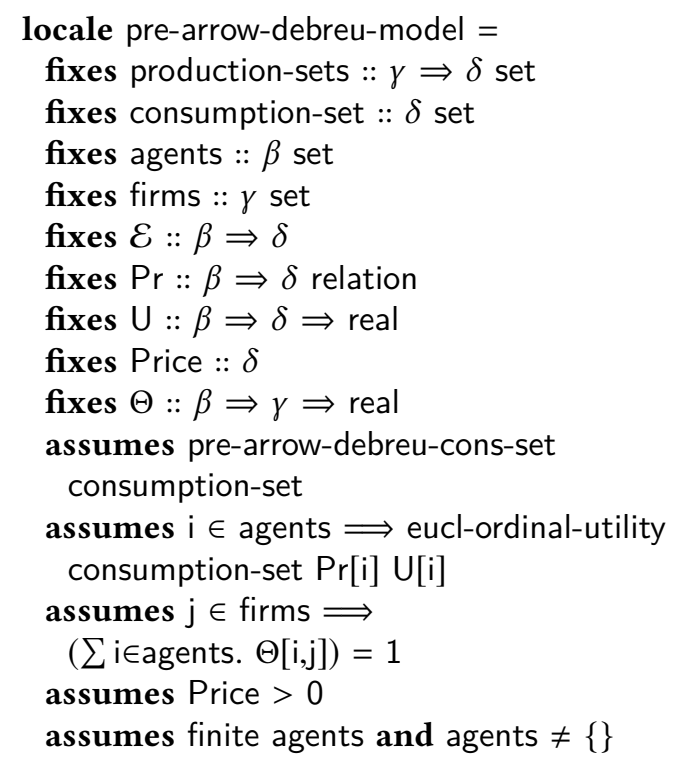

Figure 4. Locale corresponding to the constants and properties of a Private Ownership Economy.

wealth is simply comprised of the monetary value of the initial endowment. Since each agent also owns shares of some companies and receives profits from these companies accordingly, the wealth of each agent also has to account for this. Therefore, the wealth of an individual $i$ is calculated as follows:

$$
\text { Price } \bullet \mathcal{E}_{i}+\sum_{j \in f \text { firms }} \Theta_{i j} *\left(\text { Price } \bullet Y_{j}\right)
$$

where $Y_{j}$ denotes the production plan chosen by firm $j$. In the Isabelle formalization an abbreviation is used and referred to as poe-wealth. In this model the term of allocation is also extended to include both a mapping from agents to elements in the consumption set and a mapping from firms to elements in the production sets. Hence an allocation $(X, Y)$ is said to be feasible if the value of the allocation does not exceed the sum of goods produced plus the initial endowment. Moreover, all consumption and production plans need to be within their respective production and consumption sets.

Definition 10 (feasible).

feasible X Y $\longleftrightarrow\left(\sum \mathrm{i} \in\right.$ agents. $\left.\mathrm{X} \mathrm{i}\right) \leq$

$\left(\sum \mathrm{i} \in\right.$ agents. $\left.\mathcal{E}[\mathrm{i}]\right)+\left(\sum \mathrm{j} \in\right.$ firms. $\left.\mathrm{Y} \mathrm{j}\right) \wedge$

$(\forall \mathrm{i} \in$ agents. $\mathrm{X} i \in$ consumption-set $) \wedge$ 


$$
(\forall \mathrm{j} \in \text { firms. } Y \mathrm{j} \in \text { production-sets } \mathrm{j})
$$

Notice that the arguments of this predicate are $X$ and $Y$ while endowment $\mathcal{E}$ CS, and production-sets are implicit arguments provided by the context (i.e., the economy, cf. Figure4). Hence it is recommended to think of $X Y$ as a pair which together can be thought of as an allocation that allocates consumption bundles to agents and production bundles to firms. Thus, feasibility can be though of as a property of such an allocation pair .

This notion of feasibility is used in defining Pareto efficiency in this economy and therefore Definition 9 stays largely the same except that Definition 10 is used as a feasibility condition instead of 7 . However, the Walrasian equilibrium now also has to account for the addition of firms. Therefore, we need to introduce the profit maximization problem, a problem similar to the consumer problem in the previously considered economic model.

Definition 11 (profit-maximisation). profit-maximisation P S $=\arg -\max -\operatorname{set}(\lambda x . P \bullet x) S$

As with the consumer problem, we make use of the argmax function, demanding that the production plan $Y j$ chosen by firm $j$ maximizes the profits given the firm's production set $S$ (cf. Example 1) and the current price vector $P$. Extending Definition 8 by this notion gives us the Walrasian Equilibrium in the model with private ownership.

Definition 12 (competitive-equilibrium). competitive-equilibrium P X Y $\longleftrightarrow$ feasible X Y $\wedge$

$(\forall \mathrm{j} \in$ firms. $(Y \mathrm{j}) \in$ profit-maximisation $P$ $($ production-sets $\mathrm{j})) \wedge$

$(\forall \mathrm{i} \in$ agents. (X i) $\in$ arg-max-set $\mathrm{U}[\mathrm{i}]$ (budget-constraint P CS (poe-wealth i P Y)))

\section{The First Theorem of Welfare Economics}

Having defined all necessary microeconomic concepts, we will now focus on the main result. We will first explain the First Welfare Theorem in an Informal manner and then present an example with a graphical interpretation, the Edgeworth box. After that, we will state and proof preliminary lemmas in Section 6.1 before we dedicate ourselves to the formal proof of the First Welfare Theorem.

When evaluating the state of an economy one can look at it from multiple points of view. First, we can consider observing local properties for each actor and evaluating them for each agent individually. This is conveyed by the Walrasian Equilibrium where each economic actor is considered individually. On the other hand, one can consider global properties of a market such as Pareto Efficiency. This concept describes the efficiency across the entire market, while neglecting the individual properties of each actors. Theorem 3 puts these two vastly different concepts in relation.
Theorem 3 (First Theorem of Welfare Economics). Assuming locally non-satiated preferences for each agent, any allocation in combination with a price vector that forms a Walrasian Equilibrium is Pareto Efficient [1].

The First Welfare Theorem states that under fairly weak assumptions, a group of actors purely acting in their own interest will achieve an overall efficient state of the economy. And this state of all encompassing efficiency is reached even though none of the actors intended for it. This theorem is generally believed to be the mathematical formulation of Adam Smith's "Invisible Hand" [28]. Example 2 provides a visual interpretation of the First Welfare Theorem using the Edgeworth box.

Example 2 (Edgeworth Box). The Edgeworth Box (Fig. 5) presents a pure exchange economy with two goods and two agents in a graphic manner. The two goods are represented by the $\mathrm{X}$ and $\mathrm{Y}$ axis. One agent's origin is in the usual coordinate system's origin, while the others in the top right of the box. By definition, each point within this box constitutes a feasible allocation. Point $A$ for example, describes the allocation which allocates the bundle $(25,15)$ to the first and $(5,5)$ to the second agent, the sum of which, $(30,20)$ is less or equal to the total amount of the goods available. The dotted and dashed lines as well as the four black lines are called indifference curves or isoquants. The dotted indifferent curves (and the two black curves with the same orientation) arise from the first agent's preferences. Every point on each of the curves has the same utility (i.e., preference) as any other point on the same curve. As previously mentioned, it is easier to model preferences with a utility function, hence we used the Cobb-Douglas utility function ${ }^{5}$. Thus, by definition of the utility functions we not only have local non-satiation but also the stronger notion of monotonicity. Using this, we say that given an indifference curve all points to the top right of this curve are strictly preferred to points on the curve. The same holds symmetrically for the other agent. An endowment $E$ is given to both consumers. In our case the initial endowment is the same amount of goods $(15,10)$, for both consumers. Drawing the indifference curves through the endowment point results in the orange lens shape which is the set of all allocations that constitute an improvement for both parties. In other words, the area between the indifference curves that go through $E$ is the set of all Pareto dominating and attainable allocations. The theory states that the agents will exchange products for a certain price reaching an other allocation, for example $I$. $I$ is a Pareto improvement to $E$ but since there still exist feasible and dominating allocations, namely all points within $S$, we have not reached a Pareto Optimal allocation yet. The theory states that as agents keep exchanging goods the value and prices of goods will adjust

${ }^{5}$ Cobb-Douglas utility functions are functions of the form, $u(x, y):=x^{a} y^{b}$ where $a>0$ and $b>0$ [31]. 


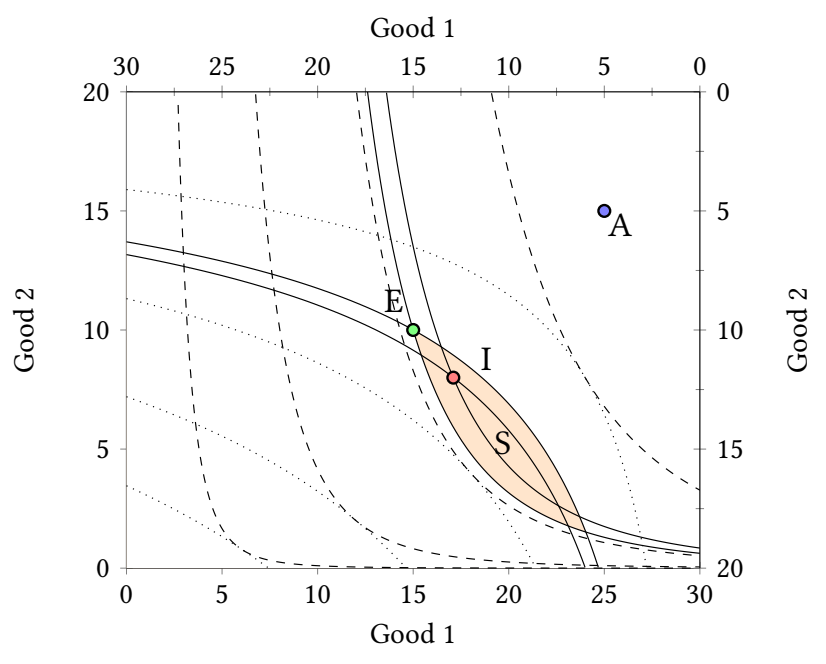

Figure 5. The Edgeworth box describing a two good two agent pure exchange economy.

until the demand equals the supply of each good, and every agent maximized their utility. At which point a Walrasian Equilibrium is reached. Furthermore, the First Welfare Theorem states, that this point is also Pareto Optimal. That is, the indifference curves going through the equilibrium allocation touch at exactly one point.

\subsection{Walras' Law and Preliminary Results}

We first show, that in a competitive equilibrium the utilities are maximized given a budget constraint. That is, all consumption bundles that are preferred must necessarily be more expensive.

Lemma 4 (all-preferred-are-more-expensive). assumes $i \in$ agents

assumes competitive-equilibrium Price $X Y$

assumes $z \in C S$

assumes $U[i] z>U[i](X i)$

shows $z \bullet$ Price $>$ Price $\bullet(X i)$

Lemma 4 follows from the definitions and properties of equilibrium and arg-max-set. The next lemma additionally requires local non-satiation since it depends on a topological argument. It basically states that if $X$ is a solution to the consumer problem, then $X$ must utilize the entire budget.

Lemma 5 (am-utilises-entire-bgt).

assumes $i \in$ agents

assumes local-nonsatiation CS $\operatorname{Pr}[i]$

and $X \in \arg$-max-set $U[i]$

(budget-constraint P CS (poe-wealth i P Y))

shows $P \bullet X=P \bullet \mathcal{E}[i]+$

$\left(\sum j \in\right.$ firms. $\left.\Theta[i, j] *(P \bullet Y j)\right)$

The proof of Lemma 5 essentially boils down to showing that the price cannot be less than the budget. This is proven by contradiction. Thus, by assuming the negation, $X$ cannot be in the bound of the budget set (cf. Definition 4). Therefore, we know that $X$ must be in the open set $\{z \in S . z<W\}$ where $S$ is the consumption set and $W$ is the wealth calculated using (3). The contradiction derives from the fact that due to local non-satiation an open set cannot have a maximum and yet $X$ is a maximum by assumption, therefore proving that $X$ must be in the bound of the budget set.

The next lemma is merely a combination of Lemmas 4 and 5 .

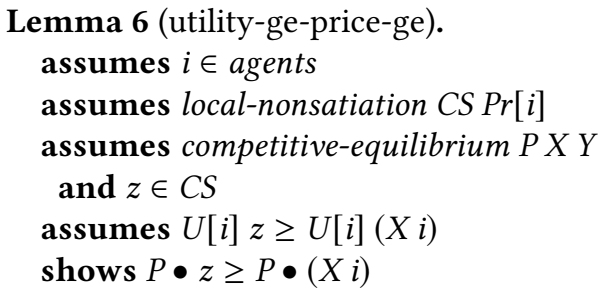

The additional assumption guarantees that the element in question $z$, is in the consumption set. The proof of Lemma 6 is based on a case distinction, where the simpler case where the utility of $z$ is strictly greater than that of $X_{i}$, can easily be proven using Lemma 4 . The case where the utilities are equal, that is the agent is indifferent towards either choice, can be shown to be true using local non-satiation and Lemma 5 .

Finally we show that Walras' law holds in our economic models.

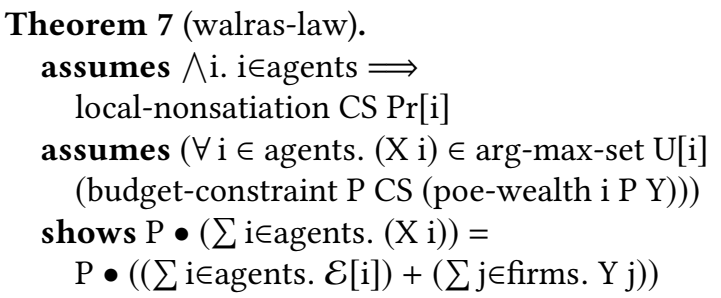

Most people are familiar with the set phrase "supply equals demand". In fact, Walras' law is the mathematical formulation thereof. This law states, that once every agent maximizes their utility, supply and demand are equal. The proof commences by showing that the sum of all budgets is completely utilized, which is the extension of Lemma 5 to the entire set of agents. Using axioms of our economic model and Lemma 5, Walras' law can be proven in few steps.

\subsection{Main Theorem}

In the previous Section we presented multiple lemmas and definitions. Now we are ready to tackle the main result, The First Welfare Theorem.

Theorem 8 (first-welfare-theorem-priv-own). assumes $\bigwedge \mathrm{i}$. $\mathrm{i} \in$ agents $\Longrightarrow$ local-nonsatiation CS $\operatorname{Pr}[\mathrm{i}]$ assumes competitive-equilibrium Price X Y shows pareto-optimal X Y 
Proof. We prove Theorem 8 by contradiction. Hence we assume the consequent to be false.

assume $\neg$ pareto-optimal $X Y$

From the Walrasian equilibrium we know that the allocation must be feasible. Recalling Definition 9 we deduce that

have $\exists X^{\prime} Y^{\prime}$. feasible $X^{\prime} Y^{\prime} \wedge X^{\prime}>$ Pareto $X$

must hold and therefore we can

obtain $X^{\prime} Y^{\prime}$ where
feasible $X^{\prime} Y^{\prime} \wedge$
$\left(\forall i \in\right.$ agents. $\left.U[i]\left(X^{\prime} i\right) \geq U[i](X i)\right) \wedge$
$\left(\exists i \in\right.$ agents. $\left.U[i]\left(X^{\prime} i\right)>U[i](X i)\right)$

We derive a contradiction by showing that $\left(X^{\prime}, Y^{\prime}\right)$ cannot be feasible. Due to the fact that all firms are profit maximizing in an Equilibrium, we know that the Inequality 4 must hold. For if it did not hold, it would contradict the fact that all firms are maximizing their profit.

$$
\text { Price } \bullet\left(\sum f \in \text { firms. } Y^{\prime} f\right) \leq \text { Price } \bullet\left(\sum j \in \text { firms. } Y j\right)
$$

Since the endowment does not change in the allocation $\left(X^{\prime}, Y^{\prime}\right)$ and we know that Theorem 7 must hold, we can deduce the following inequality:

$$
\begin{gathered}
\text { Price } \bullet\left(\left(\sum \mathrm{i} \in \text { agents. } \mathcal{E}[\mathrm{i}]\right)+\left(\sum \mathrm{f} \in \text { firms. Y f }\right)\right) \\
\geq \\
\text { Price } \bullet\left(\sum \mathrm{i} \in \text { agents. } \mathrm{X}^{\prime} \mathrm{i}\right)
\end{gathered}
$$

Using the properties of the obtained allocation $\left(X^{\prime}, Y^{\prime}\right)$ in addition with Lemmas 4 and 6 it is trivial to show that

$$
\text { Price } \bullet\left(\sum i \in \text { agents. } X^{\prime} \text { i }\right)>\text { Price } \bullet\left(\sum i \in \text { agents. X i }\right) \text {. }
$$

Furthermore, using Walras' law again the following equation must hold.

$$
\begin{gathered}
\text { Price } \bullet\left(\sum i \in \text { agents. X i }\right) \\
=
\end{gathered}
$$

$$
\text { Price } \bullet\left(\left(\sum \mathrm{i} \in \text { agents. } \mathcal{E}[\mathrm{i}]\right)+\left(\sum \mathrm{f} \in \text { firms. Y f }\right)\right)
$$

Combining the Equations 5 to 9 proves that Equation 5 must be strictly larger than 9 , a contradiction.

\subsection{The Theorem in an Exchange Economy}

In Section 6.2 we presented the statement and proof of the Welfare Theorem in the Pre-Arrow-Debreu model. The proof for the case of an exchange economy is a lot simpler and follows the same structure. We will briefly present the theorem in an exchange economy here. For more details we refer to the formalization (see conclusion).

Theorem 9 (first-welfare-theorem-exchange).

assumes $\bigwedge \mathrm{i}$. $\mathrm{i} \in$ agents $\Longrightarrow$

local-nonsatiation CS $\operatorname{Pr}[\mathrm{i}]$

assumes comp-equilib-endow Price $\mathrm{X} \mathcal{E}$

shows pareto-optimal-endow $\mathrm{X} \mathcal{E}$
In comparison with Theorem 9 the only difference are the definitions used. Namely Definition 8 is used instead of 12 . The same holds for Pareto optimality where Definition 9 is used instead of the corresponding definition in the production economy. The proof follows the same structure as the proof of Theorem 8. Furthermore, the Lemmas and Theorems 4 to 7 have equivalent counterparts in the exchange economy. The proof of this theorem is a lot shorter since the firms and their profits do not need to be accounted for.

\section{Conclusion}

In this paper, we laid out the basics of microeconomics and game theory, including the formalizations of two economic models up to the first theorem of welfare economics. We defined the basic concepts, such as preference relations and utility functions and specified properties of these. We formalized various versions of the related concepts showing the equivalences (or implications) across their presentations in the microeconomic literature. These basics have been formalized in a generic way, in order to enable further formalizations of economic theories and game theory.

The formalization consists of 1818 lines of proofs and includes 98 lemmas and theorems, as well as 24 definitions and locales. It is available at:

$$
\text { http://cl-informatik.uibk.ac.at/cek/cpp2018.tgz }
$$

with a version of the formalization already included in the Archive of Formal Proofs [23].

\subsection{Future Work}

There are plenty of directions future work could take. The concepts introduced in this theory can be used in formalizations of game theory, social choice theory, and behavioral economics. It is also possible to formalize further economic concepts including the proof of the Second Welfare Theorem and the existence of competitive equilibria. It is also possible to consider more complex models that are used to conduct economic research.

Furthermore, Game theory has multiple applications outside of economics. These include, but are not limited to, system security, cryptography, and distributed systems [22]. Since there is research in formal verification of these areas, results from formalizing (algorithmic) game theory will be of great use.

\section{Acknowledgments}

This work is supported by the Austrian Science Fund (FWF) project P26201 and the European Research Council (ERC) grant no 714034 SMART.

\section{References}

[1] Kenneth J Arrow, Amartya Sen, and Kotaro Suzumura. 2010. Handbook of Social Choice and Welfare. Vol. 2. Elsevier. 
[2] Clemens Ballarin. 2014. Locales: A Module System for Mathematical Theories. Fournal of Automated Reasoning 52, 2 (01 Feb 2014), 123-153. https://doi.org/10.1007/s10817-013-9284-7

[3] Lawrence Blume, David Easley, Jon Kleinberg, Robert Kleinberg, and Éva Tardos. 2015. Introduction to computer science and economic theory. Fournal of Economic Theory 156, Supplement C (2015), 1 13. https://doi.org/10.1016/j.jet.2014.11.002 Computer Science and Economic Theory.

[4] Marco B. Caminati, Manfred Kerber, Christoph Lange, and Colin Rowat. 2014. Set Theory or Higher Order Logic to Represent Auction Concepts in Isabelle? Springer International Publishing, Cham, 236-251. https: //doi.org/10.1007/978-3-319-08434-3_18

[5] John C. Cox, Stephen A. Ross, and Mark Rubinstein. 1979. Option pricing: A simplified approach. Fournal of Financial Economics 7, 3 (1979), 229 - 263. https://doi.org/10.1016/0304-405X(79)90015-1

[6] Manuel Eberl. 2016. Randomised Social Choice Theory. Archive of Formal Proofs (May 2016). http://isa-afp.org/entries/Randomised_ Social_Choice.html, Formal proof development.

[7] Mnacho Echenim and Nicolas Peltier. 2017. The Binomial Pricing Model in Finance: A Formalization in Isabelle. In Automated Deduction - CADE 26 - 26th International Conference on Automated Deduction, Gothenburg, Sweden, August 6-11, 2017, Proceedings (Lecture Notes in Computer Science), Leonardo de Moura (Ed.), Vol. 10395. Springer, 546562. https://doi.org/10.1007/978-3-319-63046-5 33

[8] Benjamin Edelman, Michael Ostrovsky, and Michael Schwarz. 2007. Internet Advertising and the Generalized Second-Price Auction: Selling Billions of Dollars Worth of Keywords. American Economic Review 97, 1 (March 2007), 242-259. https://doi.org/10.1257/aer.97.1.242

[9] Peter Gammie. 2008. Some classical results in Social Choice Theory. Archive of Formal Proofs (Nov. 2008). http://isa-afp.org/entries/ SenSocialChoice.html, Formal proof development.

[10] John Geanakoplos. 1989. Arrow-Debreu Model of General Equilibrium. Palgrave Macmillan UK, London, 43-61. https://doi.org/10.1007/ 978-1-349-19802-3 3

[11] Philip J. Reny Geoffrey A. Jehle. 2011. Advanced Microeconomic Theory (3 ed.). Pearson Education Limited.

[12] Florian Haftmann and Makarius Wenzel. 2007. Constructive Type Classes in Isabelle. Springer Berlin Heidelberg, Berlin, Heidelberg, 160-174. https://doi.org/10.1007/978-3-540-74464-1_11

[13] John Harrison, Josef Urban, and Freek Wiedijk. 2014. History of Interactive Theorem Proving. In Computational Logic, Jörg H. Siekmann (Ed.). Handbook of the History of Logic, Vol. 9. Elsevier, 135-214. https://doi.org/10.1016/B978-0-444-51624-4.50004-6

[14] Peter Jaeger. 2012. Elementary Introduction to Stochastic Finance in Discrete Time. Formalized Mathematics 20, 1 (2012), 1-5. http: //eudml.org/doc/267949

[15] Donald W. Katzner. 2006. An Introduction to the Economic Theory of Market Behavior: Microeconomics from a Walrasian Perspective. Edward Elgar Publishing Limited.

[16] Manfred Kerber, Christoph Lange, Colin Rowat, and Wolfgang Windsteiger. 2013. Developing an Auction Theory Toolbox. AISB 2013 (2013), 1-4.

[17] Alan Kirman. 2010. The Economic Crisis is a Crisis for Economic Theory*. CESifo Economic Studies 56, 4 (2010), 498-535. https://doi. org/10.1093/cesifo/ifq017

[18] David M. Kreps. 1990. A Course in Microeconomic Theory. Princeton University Press. https://books.google.at/books?id=nN1bPwAACAAJ
[19] Christoph Lange, Colin Rowat, and Manfred Kerber. 2013. The ForMaRE Project - Formal Mathematical Reasoning in Economics. In Intelligent Computer Mathematics - MKM, Calculemus, DML, and Systems and Projects 2013, Held as Part of CICM 2013, Bath, UK, Fuly 8-12, 2013. Proceedings (Lecture Notes in Computer Science), Jacques Carette, David Aspinall, Christoph Lange, Petr Sojka, and Wolfgang Windsteiger (Eds.), Vol. 7961. Springer, 330-334. https://doi.org/10.1007/ 978-3-642-39320-4 23

[20] Andreu Mas-Colell, Michael Whinston, and Jerry Green. 1995. Microeconomic Theory. Oxford University Press. http://EconPapers.repec. org/RePEc:oxp:obooks:9780195102680

[21] Tobias Nipkow. 2008. Arrow and Gibbard-Satterthwaite. Archive of Formal Proofs (2008). https://www.isa-afp.org/entries/ ArrowlmpossibilityGS.shtml

[22] Noam Nisan, Tim Roughgarden, Eva Tardos, and Vijay V. Vazirani. 2007. Algorithmic Game Theory. Cambridge University Press, New York, NY, USA.

[23] Julian Parsert and Cezary Kaliszyk. 2017. Microeconomics and the First Welfare Theorem. Archive of Formal Proofs 2017 (2017). https: //www.isa-afp.org/entries/First_Welfare_Theorem.html

[24] Robert Pindyck and Daniel Rubinfeld. 2012. Microeconomics (8th Edition) (The Pearson Series in Economics). Pearson.

[25] Stéphane Le Roux. 2009. Acyclic Preferences and Existence of Sequential Nash Equilibria: A Formal and Constructive Equivalence. In Theorem Proving in Higher Order Logics, 22nd International Conference, TPHOLs 2009, Munich, Germany, August 17-20, 2009. Proceedings (Lecture Notes in Computer Science), Stefan Berghofer, Tobias Nipkow, Christian Urban, and Makarius Wenzel (Eds.), Vol. 5674. Springer, 293-309. https://doi.org/10.1007/978-3-642-03359-9_21

[26] Adam Smith. 1776. An Inquiry Into the Nature and Causes of the Wealth of Nations. Number v. 1 in An Inquiry Into the Nature and Causes of the Wealth of Nations. Strahan. https://books.google.at/books?id= C5dNAAAAcAAJ

[27] Ross M. Starr. 2011. General Equilibrium Theory: An Introduction. Cambridge University Press. https://books.google.at/books? id $=$ t9pSAIEMyDYC

[28] Joseph E. Stiglitz. 1991. The Invisible Hand and Modern Welfare Economics. Working Paper 3641. National Bureau of Economic Research. https://doi.org/10.3386/w3641

[29] Joseph E. Stiglitz. 2011. Rethinking Macroeconomics: What failed, and how to repair it. Journal of the European Economic Association 9, 4 (2011), 591-645. https://doi.org/10.1111/j.1542-4774.2011.01030.x

[30] Steven Tadelis. 2013. Game Theory: An Introduction. Princeton University Press.

[31] Hal R. Varian. 2014. Intermediate Microeconomics: A Modern Approach (9th ed.). W. W. Norton \& Co Inc.

[32] John von Neumann, Oskar Morgenstern, Harold W. Kuhn, and Ariel Rubinstein. 1944. Theory of Games and Economic Behavior (60th Anniversary Commemorative Edition). Princeton University Press. http://www.jstor.org/stable/j.ctt1r2gkx

[33] Makarius Wenzel, Lawrence C. Paulson, and Tobias Nipkow. 2008. The Isabelle Framework. In Theorem Proving in Higher Order Logics, 21st International Conference, TPHOLs 2008 (LNCS), Otmane Aït Mohamed, César A. Muñoz, and Sofiène Tahar (Eds.), Vol. 5170. Springer, 33-38. https://doi.org/10.1007/978-3-540-71067-7_7

[34] Freek Wiedijk. 2009. Formalizing Arrow's theorem. Sadhana 34, 1 (01 Feb 2009), 193-220. https://doi.org/10.1007/s12046-009-0005-1 